\title{
Globalisation et espaces locaux
}

Margarita ESTRADA IGUínIZ et Pascal LABAZÉE (coord.), Globalización y localidad : espacios, actores, movilidades e identidades, Mexico, Publicaciones de la Casa Chata, Centro de Investigación y Estudios Superiores en Antropología social (CIESAS) et Institut de recherche pour le développement, 2007, $625 \mathrm{p}$.

\section{Évelyne Mesclier}

\section{OpenEdition}

\section{Journals}

Édition électronique

URL : http://journals.openedition.org/transcontinentales/922

DOI : $10.4000 /$ transcontinentales.922

ISBN : 978-2-7351-1561-7

ISSN : 1775-397X

\section{Éditeur}

Editions de la maison des sciences de l'homme

\section{Édition imprimée}

Date de publication : 30 juin 2008

Pagination : 156-158

ISBN : 978-2-200-92511-6

ISSN : 1950-1684

Référence électronique

Évelyne Mesclier, «Globalisation et espaces locaux », Transcontinentales [En ligne], 6 | 2008, document 14, mis en ligne le 06 avril 2011, consulté le 25 septembre 2020. URL : http://journals.openedition.org/ transcontinentales/922; DOI : https://doi.org/10.4000/transcontinentales.922 
The Karakorum Highway). Le transit entre le Xinjiang et le nord du Pakistan peut aussi avoir des effets moins recherchés par Pékin lorsqu'il s'agit du soutien d'une militance islamiste aux séparatistes ouïgours (Abanti Bhattacharya, The Xinjiang Factor).

On peut seulement regretter que le délai entre la remise des contributions et la publication n'ait pas été plus court : cet intervalle souvent important dans les travaux collectifs, est plus encore dommageable sur des questions d'actualité. Face au parti pris d'avoir un grand nombre d'auteurs (18), des coupes auraient aussi été souhaitables afin d'éviter nombre de redites. On aurait également aimé que la relation bilatérale soit parfois replacée dans une perspective plus globale. Il est souvent écrit par exemple que le Pakistan représente pour la Chine un point d'entrée dans le monde musulman, mais sans donner des manifestations concrètes de cette attente. Cet ouvrage n'en offre pas moins un panorama quasi exhaustif des multiples facettes de la coopération stratégique sino-pakistanaise telle qu'elle est perçue par les analystes indiens. Il comble à cet égard un vide.

Gilles Boquérat, chercheur résident à l'Institut d'études stratégiques d'Islamabad, chercheur associé au Centre d'études de l'Inde et de l'Asie du Sud (CNRS-EHESS), Paris
Globalisation et espaces locaux

Margarita Estrada IguínIZ et Pascal Labazée (coord.), Globalización y localidad: espacios, actores, movilidades e identidades, Mexico, Publicaciones de la Casa Chata, Centro de Investigación y Estudios Superiores en Antropología social (CIESAS) et Institut de recherche

pour le développement, 2007, 625 p.

Cet ouvrage, épais volume de plus de 600 pages, explore les liens entre la globalisation et les espaces locaux. La plupart des vingt et quelque contributions portent sur le Mexique, le plus souvent sur l'État de Guanajuato, mais quelques-unes présentent des études de cas réalisées au Brésil, en Argentine ou à Porto Rico, voire en Inde ou au Mali. Dans l'ensemble, disons-le d'emblée, ce choix emporte l'adhésion: la confrontation d'observations centrées sur un terrain particulier à des éclairages, même rapides, sur d'autres parties du monde permet effectivement de mieux saisir la dimension globale des évolutions en cours, tout en soulignant les particularités locales.

L'ouvrage est composé d'un prologue, d'une introduction et de quatre sections, autour des espaces productifs, des acteurs sociaux, des mobilités et du rapport entre travail et identité. La distribution des textes pose parfois question. À première lecture, la reconfiguration des espaces ruraux du Veracruz semble être aussi bien le pendant des recompositions industrielles des villes du Guanajuato qu'une affaire de migrations; les textes portant sur les migrants sont classés parfois du côté de la réflexion sur les acteurs sociaux, parfois de la description des mobilités. Les auteurs préviennent cependant la critique dès l'introduction : leur objectif est précisément de montrer l'interdépendance des quatre thèmes. Le lecteur est effectivement convié à reconstituer depuis plusieurs angles de vue, en 
décalage plus ou moins important les uns avec les autres, un même phénomène aux facettes multiples. Le bon niveau général des contributions, dont seules quelquesunes échouent à cerner clairement leur objet et son lien avec la thématique générale, et la qualité de l'édition, permettent de bien suivre le fil directeur.

Les conclusions auxquelles nous conduit l'ouvrage sont riches. Elles remettent en cause un certain nombre d'idées reçues, à partir d'exemples précis ou de réflexions plus théoriques mais également étayées par le terrain. Ainsi, plusieurs contributeurs démontrent que la concentration spatiale d'entreprises spécialisées ne suffit pas à garantir leur adaptation à l'augmentation de la concurrence, dans une globalisation définie comme le passage d'une économie d'échanges entre puissances nationales à des transactions directes entre espaces infranationaux. Le capital social des entrepreneurs est crucial, la coordination entre les acteurs ne va par ailleurs pas toujours de soi, enfin les configurations productives et leurs évolutions sont plus diverses que ne le laisseraient penser les modèles théoriques. Est remise en cause également l'idée selon laquelle la décentralisation suffirait à permettre aux municipalités d'orienter le changement économique local : celui-ci est déterminé souvent bien loin de leur sphère d'influence.

Au-delà de la "globalisation» des échanges, la "mondialisation" est aussi faite de l'accélération des circulations en tous genres. L'ouvrage montre que les migrations, phénomène ancien s'il en est, prennent de nouvelles formes spatiales et entraînent des dynamiques sociales originales. Les migrants prennent conscience de leur lien avec leur propre État national, qui défend leur statut à l'étranger, mais, observant le fonctionne- ment de sociétés où le citoyen est mieux reconnu, revendiquent aussi une amplification de leurs droits. Les attaches avec la localité d'origine, la constitution de "clubs » à l'étranger, aspects plus souvent étudiés, prennent des tours inédits avec la décentralisation et l'intervention des migrants dans la politique locale. Il ne faut pas pour autant conclure que le changement ne touche que les migrants. L'ouvrage s'élève en particulier contre l'idée de l'existence de "territoires ethniques» qui seraient par contraste figés, et souligne la complexité des configurations. Il montre que des choix sont faits à chaque instant, d'adopter par exemple des modes de consommation alimentaire importés, mais aussi de ne pas rejoindre le conjoint aux États-Unis ou de privilégier l'éducation des filles, plus susceptibles de rester. Il décrit enfin comment la perte d'un emploi salarié stable, fréquente dans la nouvelle donne économique, fait s'interroger sur leur identité des hommes et des femmes de tous les milieux sociaux : à la perte de revenus, souvent définitive, s'ajoute le sentiment de déchéance sociale associé à l'auto-emploi ou au chômage.

La fin de l'ouvrage laisse le lecteur sur la note plutôt noire de la précarisation et de la démoralisation de populations confrontées à la restructuration du monde du travail. On peut regretter l'absence de conclusion qui reprendrait l'ensemble des facettes évoquées. Il faut saluer parallèlement la clarté des propos introductifs des deux coordinateurs. C'est en effet, paradoxalement, dans l'introduction qu'il faut chercher la présentation des problématiques abordées, mais aussi des résultats de recherche exposés dans les contributions.

Dans le prologue, Claude Bataillon, depuis une trajectoire professionnelle 
qui couvre plusieurs décennies, rappelle pourquoi et comment les scientifiques en sont venus à s'intéresser aux échelles infranationales de la réalité mexicaine, et quels sont les défis auxquels ont à répondre les études sur le local dans le contexte de la globalisation. Ces défis paraissent ici bien relevés. Malgré quelques contributions plus faibles, lot presque inévitable d'un travail collectif, l'ouvrage présente le grand intérêt d'allier des questionnements théoriques à la présentation d'abondants résultats de terrain, qui permettent d'avancer dans la réflexion sur un sujet fondamental de par ses conséquences sociales.

Évelyne Mesclier, chercheur de l'Institut de recherche pour le développement (IRD), associée à I'UMR CNRS PRODIG

(Pôle de recherche pour l'organisation et la diffusion de l'information géographique) 